\title{
Das Quartärprofil von Hagelstadt im Bayerischen Tertiärhügelland
}

\author{
HORST STRUNK *)
}

\author{
Sections, Upper Pleistocene, lithostratigraphy, Wurm, Riss/Wurm Interglacial, \\ paleosols, luvisols, grey forest soils, pseudogleys \\ Bavaria, Alpenvorland, TK 7139
}

\begin{abstract}
Kurzfassung: Am Nordrand des Bayerischen Tertiärhügellandes liegt in der Nähe von Regensburg das mächtige Quartärprofil der Ziegeleigrube von Hagelstadt, mit seinen acht Böden interglazialen Typs das vielgliedrigste Lößprofil Südbayerns. Die fossilen Böden sind Parabraunerden bzw. Pseudogley-Parabraunerden, die aus Lössen und Staublehmen hervorgingen, welche in den pleistozänen Kaltzeiten am Rand eines Plateaus über dem Donautal abgelagert wurden. Geologischer und pedologischer Aufbau des Profils werden eingehend beschrieben und dessen Einordnung in die Pedostratigraphie Mitteleuropas versucht.
\end{abstract}

[The Quaternary Profile of Hagelstadt in The Bavarian Tertiary Molasse-Hills]

\begin{abstract}
On the northern margin of the Bavarian Tertiary Molasse-Hills one can find the brickyard of Hagelstadt in the vicinity of Regensburg, with its thick Quaternary profile. Consisting of eight soils of interglacial character it represents the best differentiated loess-profile of southern Bavaria. Its fossilized soils - orthic, chromic, and gleyic luvisols developed from wind-deposited loesses or dustloams on the margin of a plateau above the Danube valley. The profile's geological and pedological structure is described in detail; and there is a tentative chronological classification within the pedostratigraphy of Central Europe.
\end{abstract}

\section{Einleitung}

Etwa $18 \mathrm{~km}$ südöstlich des Donauknies bei Regensburg liegt am Nordrand des Bayerischen Tertiärhügellandes die Ziegeleigrube von Hagelstadt. Seit mehreren Jahren ist dort ein siebzehn Meter mächtiges, vielgliedriges Quartärprofil aufgeschlossen, welches die vollständigste Löß-Paläoböden-Abfolge Südbayerns sein dürfte. In seiner Gesamtlänge von einhundertzwanzig Metern wurde dieses Profil in den Jahren

*) Anschrift des Verfassers: Dr. H. StrunK, Geographisches Institut der Universität Regensburg, Universitätsstr. $31, \mathrm{D}-8400$ Regensburg.
1984 und 1985 von einer Arbeitsgruppe des Geographischen Instituts der Universität Regensburg aufgenommen und beprobt. Weitere sechs Profilmeter wurden 1986 unter der Grubensohle in Fortsetzung von Profil Nr. 392 bis in die liegenden Quarzkiese erbohrt und beprobt. Durch den fortschreitenden Abbau ergaben sich seitdem nur noch geringe Modifizierungen im Profilaufbau, die uns die stratigraphische Gliederung laufend zu verfeinern halfen. Diese wurde durch Bohrprofile in nördlicher und westlicher Richtung ergänzt, wodurch erst die räumliche Vorstellung von der Verbreitung und dem Erhaltungszustand der zahlreichen Paläoböden ermöglicht wurde.

Mit seinen acht Böden interglazialen Typs übertrifft das Profil Hagelstadt die schon früher aus den benachbarten, mittlerweile aufgelassenen Ziegeleigruben in Hagelstadt von BRUNNACKER (1964) und Köfering (BRUNNACKer 1957) beschriebenen Profile erheblich. Die Lage dieser mittlerweile verstürzten Profile geht aus Abb. 1 hervor. Auffallende pedologische Ähnlichkeit weist das Profil Hagelstadt mit dem leider ebenfalls verstürzten Quartärprofil in der ehemaligen Ziegeleigrube Strobel im Regental unmittelbar nördlich von Regensburg auf, das sieben Böden interglazialen Typs und damit nur einen weniger als das Profil Hagelstadt aufweist (BRUNNACKER 1964; BRUNNACKER et al. 1976). Hinsichtlich der geologisch-tektonischen und auch der topographischen Situation bestehen zwischen diesen beiden Profilen jedoch erhebliche Differenzen, die einen genetischen Vergleich erschweren.

\section{Geologischer Überblick}

Im Regensburger Raum grenzen drei geologische Einheiten aneinander, im NE der Falkensteiner Vorwald als Südwestteil des altkristallinen Moldanubischen Grundgebirges, im N und $\mathrm{W}$ die aus jurassischen und kretazischen Gesteinen aufgebaute Fränkische Alb 


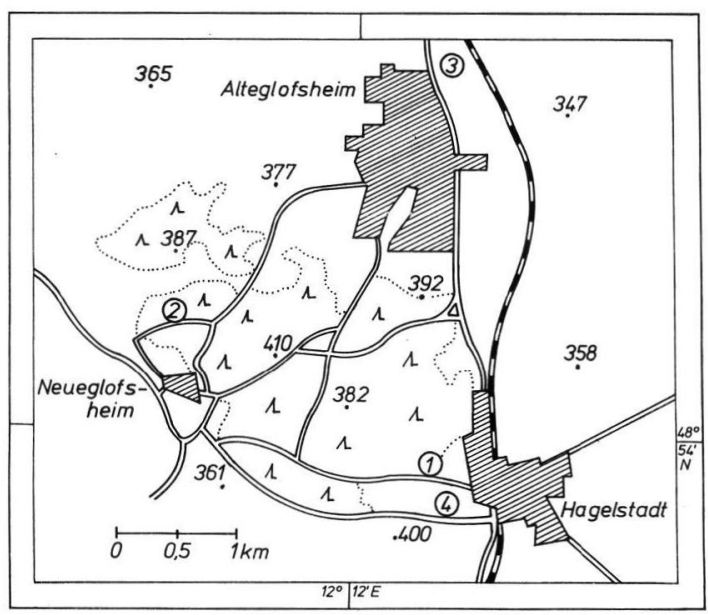

Abb. 1: Die Lage der Bodenprofile im Raum Hagelstadt: 1 = heutiges Profil in der Ziegeleigrube Hagelstadt; 2 = Profil Sandgrube Neueglofsheim (STRUNK 1989); 3 = Profil Ziegeleigrube Köfering (BRUNNACKER 1957); 4 = Profil Ziegeleigrube Hagelstadt (BRUNNACKER 1964). Höhenpunkte in $m$ ü. NN.

und im S das flachwellige Niederbayerische Tertiärhügelland am Nordrand des tertiären Molassebeckens im Alpenvorland. Hauptvorfluter ist die Donau, die das Gebiet annähernd von W nach $E$ quert. Die Ziegeleigrube von Hagelstadt liegt mit ihrer Oberkante auf $391 \mathrm{~m} \mathrm{NN}$ rund $25 \mathrm{~m}$ höher als die lößbedeckte, vom Langenerlinger Bach zerschnittene Terrassenfläche des Älteren Deckenschotters der Donau (LEGER 1965: WeINIG 1980). Allein die pleistozänen Lösse und Lößlehme können in N- bis E-exponierten Leelagen am Rand der breiten Höhenrücken 20-25 m mächtig werden (WEINIG 1980; HomILIUS et al. 1983); größere und tiefere Aufschlüsse sind leider sehr selten. Diese überwiegend schluffigen Sedimente des Quartärs überlagern mächtige tertiäre Tone und schluffige Feinsande der Oberen Süßwassermolasse, an deren Top ein 10-15 m mächtiger Quarzschotter eingeschaltet ist, der sogenannte "Höhenhofer Schotter”.

Abb. 2: Das Quartärprofil in der Ziegeleigrube Hagelstadt (Abbaustand Herbst 1985) mit der Lage der beprobten Schlitzprofile Nr. 391 und Nr. 392. Die Horizontnummern entsprechen denen in $\mathrm{Abb}$. 3 und $\mathrm{Abb} .4: 1=\mathrm{Al}$-Horizont; 2 = Schluff; 3 = Lehm; 4 = Verbraunung (Bv-Horizont); $5=$ Parabraunerde (Bt-Horizont);

$6=$ Pseudogley-Parabraunerde, schwach/stark vergleyt .

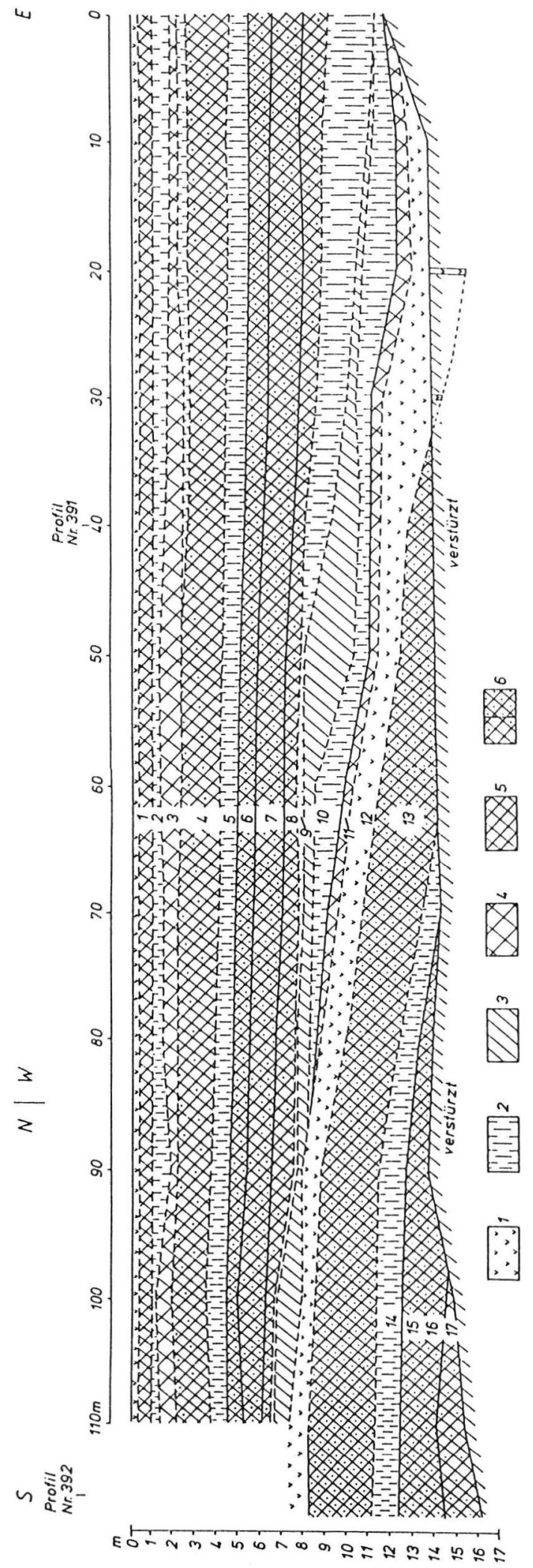




\section{Das Profil Hagelstadt}

Die Ziegeleigrube von Hagelstadt liegt am Westrand dieses etwa $18 \mathrm{~km}$ südöstlich von Regensburg gelegenen Dorfes (vgl. Abb. 1), am Ostrand eines zungenförmig nach $\mathrm{E}$ vorspringenden, nahezu ebenen Plateaus mit Höhen zwischen $390 \mathrm{~m}$ und $395 \mathrm{~m} \mathrm{NN}$. Nach Ergebnissen refraktionsseismischer Messungen des Verf. sind die quartären Lößlehme dort rund $26 \mathrm{~m}$ mächtig und werden, unterbrochen von einem geringmächtigen Horizont sandigen Schotters, von kompakten, graublauen Tonen der Oberen Süßwassermolasse unterlagert. Aufgeschlossen sind im Profil nur die oberen $17 \mathrm{~m}$ dieser Abfolge. Die Gesamtsituation ist in Abb. 2 dargestellt (Abbaustand 1985). Da die Grube den Westhang einer nach $\mathrm{N}$ bis NE ziehenden ehemaligen Tiefenlinie anschneidet, deren damalige Oberfläche von den Horizonten 12 und 13 (5. fBtSd) nachgezeichnet wird, tauchen nach $S$ immer ältere Horizonte über dem Grubenplanum auf, während am Ostrand der Grube die jüngeren Horizonte ihre größte Mächtigkeit und Differenzierung erreichen.

\subsection{Horizontverzeichnis}

Die vollständige Horizontabfolge wurde in zwei Schlitzprofilen beprobt, und zwar Profil Nr. 391 in

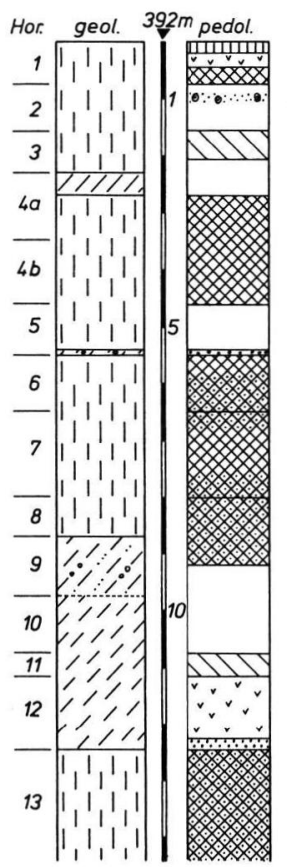

Holozäne Parabraunerde Graubrauner Nafboden, leicht rostfleckig, mit Pupilla sp.

$f B v$ des Mittelwürm

1. $f B t$

Mn-Konkretionen

2. $f B t S d$

3. $f B t S d$

4. $f B t S d$

$f B v$

Fossiler autochthoner - parautochthoner Al-AlSw-Horizont

Anreicherung von Mn-Konkretionen

5. $f B t S d$

Abb. 3: Ziegeleigrube Hagelstadt, Nordwand, Profil Nr. 391. Die Horizontnummern entsprechen denen in Abb. 2. Signaturlegende wie in Abb. 7. der Mitte der Gruben-Nordwand (vgl. Abb. 3), die tieferen Horizonte zusätzlich in Profil Nr. 392 aus der Westwand (vgl. Abb. 4), an deren Fuß die nicht aufgeschlossenen Horizonte unter der Grubensohle bis in den liegenden Schotter erbohrt wurden. Diese Profile wurden zusammen mit Herrn Dr. BUCH aufgenommen. Granulometrie, pH-Werte, Kalkgehalte und Munsell-Farben sind in Abb. 5 dargestellt, die Signaturlegenden weiter hinten in $\mathrm{Abb} .7$.

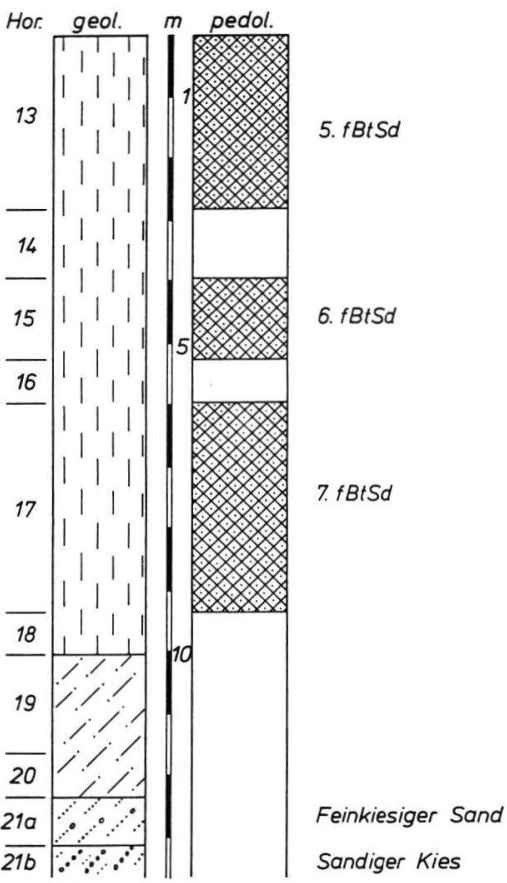

Abb. 4: Ziegeleigrube Hagelstadt, Westwand, Profil Nr. 392. Die Horizontnummern entsprechen denen in Abb. 2. Signaturlegende wie in Abb. 7.

Profil Nr. 391, Gruben-Nordwand (H 5417820, R $4515320 ; 391 \mathrm{~m}$ üb. NN)

\section{Horizont Mächtigkeit}

$0,1 \mathrm{~m}$ Schluff, humos, gelblich braun (10 YR 5/4)

$0,3 \mathrm{~m} \quad$ Schluff, gelblich braun (10 YR 5/6), leicht humos, unteres Drittel leicht graufleckig mit winzigen $\mathrm{Fe}-/ \mathrm{Mn}$-Konkretionen

$0,3 \mathrm{~m} \quad$ schluffig-toniger Lehm, kräftig braun (7,5 YR 3/4), mit scharfer Untergrenze übergehend in

0,8 m Löß, gelbbraun (10 YR 6/5), carbonatreich, mit Pseudomycelien, an der Basis mit Kalkkonkretionen $-1 \mathrm{~cm} \varnothing$, zahlreiche Mollusken im oberen Drittel 

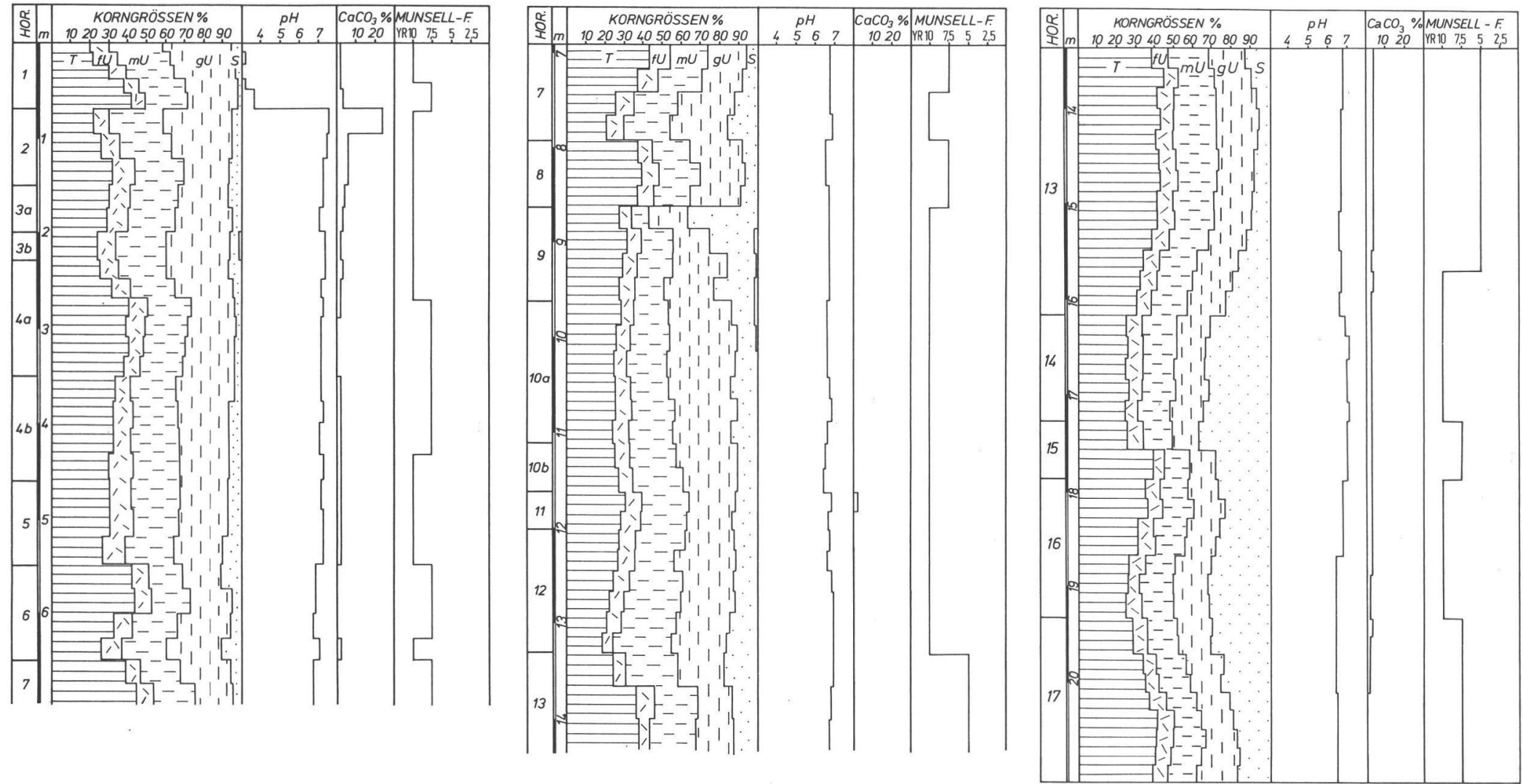

Abb. 5: Granulometrie, pH-Werte, Kalkgehalte und Munsell-Farben der beprobten Horizonte des Profils Hagelstadt. 
Horizont Mächtigkeit

$0,5 \mathrm{~m}$

$3 a$

\begin{tabular}{|c|c|c|}
\hline $3 b$ & $0,3 \mathrm{~m}$ & Löß, gelbbraun (10 YR 6/5), car- \\
\hline
\end{tabular}

$0,4 \mathrm{~m}$ schluffiger Lehm, bräunlich gelb (10 YR 6/6), leicht pseudovergleyt, schwach $\mathrm{Fe}-/ \mathrm{Mn}$-fleckig, Lößkindel,

$\mathrm{Fe}-/ \mathrm{Mn}-$ Konkretionen und zahlrei-

$4 \mathrm{a}$

che Holzkohleflitter an der Basis (Basisfließerde)

$0,8 \mathrm{~m} \quad$ schluffig-toniger Lehm bis schluffiger Ton, braun (7,5 YR 4/4), ohne Pseudovergleyung und Fe-/MnFleckung; vereinzelt Lößkindel an der Basis

$1,1 \mathrm{~m}$ schluffiger Lehm, im oberen Teil braun (7,5 YR 4/4), nach unten gelbbraun (10 YR 6/4), obere $0,2 \mathrm{~m}$ schwach $\mathrm{Fe}-/ \mathrm{Mn}$-fleckig

$4 b$

$0,9 \mathrm{~m}$ schluffiger Lehm, hell gelbbraun (10 YR 6/4), carbonatfrei, nadelstichporig, in Poren Tonhäutchen, an der Basis $10 \mathrm{~cm}$ mächtiger $\mathrm{Fe}-1$ Mn-Konkretionshorizont (Konkretionen bis $1 \mathrm{~cm} \varnothing$ )

$1,0 \mathrm{~m}$ schluffig-toniger Lehm, obere $30 \mathrm{~cm}$ kräftig braun (7,5 YR 4/6), untere $70 \mathrm{~cm}$ stark pseudovergleyt, gelblich marmoriert; an der Basis $10 \mathrm{~cm}$ mächtiger Horizont von Fe- 1 Mn-Konkretionen bis $1 \mathrm{~cm} \varnothing$

$1,5 \mathrm{~m}$ toniger Lehm, obere $30 \mathrm{~cm}$ pseudovergleyt, gelbgrau marmoriert, davon obere $10 \mathrm{~cm}$ mit $\mathrm{Fe}$ - $/ \mathrm{Mn}$ Konkretionen, untere $70 \mathrm{~cm}$ braun (7,5 YR 4/4), auf Leitbahnen pseudovergleyt, Mn-Ausscheidungen auf den Gefügeflächen; untere $50 \mathrm{~cm}$ stark pseudovergleyt, $\mathrm{Fe}-1$ Mn-Konkretionshorizont mit Konkretionen bis $0,5 \mathrm{~cm} \varnothing$ an der Basis

$0,7 \mathrm{~m} \quad$ schluffig-toniger Lehm, braun (7,5 YR 4/4), auf Leitbahnen pseudovergleyt, obere $10 \mathrm{~cm}$ schwach Fe-/ Mn-fleckig

$1,0 \mathrm{~m}$ schluffiger Lehm, sand- und kiesstreifig, kräftig braun (7,5 YR 5/8), auf Leitbahnen pseudovergleyt, auf den unteren $50 \mathrm{~cm}$ gelblich braun (10 YR 5/8) mit Fe-/ Mn-Ausfällungen im Bereich der Kiesschnüre (Umlagerungshorizont, kappt nach E das Profil bis zur Grubensohle)
Horizont Mächtigkeit

$1,5 \mathrm{~m}$ schluffiger Lehm, stark pseudovergleyt, bräunlich gelb (10 YR 6/6), grau marmoriert, durchgehend Fe-/Mn-fleckig

\begin{tabular}{ll}
$\frac{10 \mathrm{~b}}{11}$ & $\begin{array}{c}0,5 \mathrm{~m} \\
0,4 \mathrm{~m} \\
1,3 \mathrm{~m}\end{array}$ \\
\hline 12 & $1,0 \mathrm{~m}$
\end{tabular}

schluffiger Lehm, gelbbraun (10 YR 5/6), schwach Fe-/Mn-fleckig schluffig-toniger Lehm, gelbbraun (10 YR 5/6), Scherbengefüge

schluffiger Lehm, stark Fe-/Mnfleckig; obere $30 \mathrm{~cm}$ bräunlich gelb (10 YR 6/6), dann gelb (10 YR 7/6), dann übergehend in gelbgrau, reduziert auf den Gefügeflächen; bis $0,2 \mathrm{~m}$ mächtige Konzentration von $\mathrm{Fe}-/ \mathrm{Mn}$-Konkretionen bis $2 \mathrm{~cm} \varnothing$ an der Basis

toniger Lehm, gelblich rot (5 YR 5/6), grau marmoriert, stark pseudovergleyt, starke $\mathrm{Fe}$-/Mn-Ausfällungen auf den Gefügeflächen, $\mathrm{Fe}-/ \mathrm{Mn}$-Konkretionen bis $2 \mathrm{~cm} \varnothing$ im gesamten Profil verteilt; im Liegenden übergehend in gelblich roten, tonigen Lehm

Profil Nr. 392, Gruben-Westwand (H 5417800, R $4515210 ; 380 \mathrm{~m}$ üb. NN)

$2,8 \mathrm{~m}$ toniger Lehm, gelblich rot (5 YR 5/6), grau marmoriert, stark pseudovergleyt, starke Fe-/Mn-Ausfällungen auf den Gefügeflächen, übergehend in

$1,1 \mathrm{~m}$ sandiger Lehm, gelbbraun (10 YR 5/7), graufleckig auf den Gefüge-
14

15

\section{$0,6 \mathrm{~m}$}

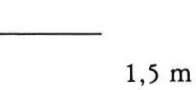

16

$17 \quad 1,7 \mathrm{~m}$ flächen, in der unteren Hälfte vereinzelte $\mathrm{Fe}-/ \mathrm{Mn}$-Konkretionen bis $1 \mathrm{~cm} \varnothing$, übergehend in

toniger Lehm, kräftig braun $(7,5$ YR 5/8), leicht graufleckig, auf den oberen $20 \mathrm{~cm}$ sehr stark, darunter stark mit Fe-/Mn-Konkretionen bis $1,5 \mathrm{~cm} \varnothing$ durchsetzt; Konkretionen nach unten zu abnehmend, übergehend in

YR 6/6), stark graufleckig, verein zelt kleine $\mathrm{Fe}-/ \mathrm{Mn}$-Konkretionen, übergehend in

leicht sandiger, toniger Lehm, kräftig baun (7,5 YR 4/6), graufleckig, stark durchsetzt mit Nestern von kleinen Fe-/Mn-Konkretionen, diese ab 1,2 $\mathrm{m}$ nach unten wieder abnehmend (Grubensohle) 
Bohrung am Profilfuß von Nr. 392

17

$$
\begin{aligned}
& 1,7 \mathrm{~m} \\
& 0,7 \mathrm{~m}
\end{aligned}
$$

18

$1,6 \mathrm{~m}$ schluffiger Lehm, leicht feinsandig, hell gelblich braun $(2,5 \mathrm{Y}$ 6/4), grau marmoriert, im unteren Drittel zunehmend sandstreifig, übergehend in

$0,7 \mathrm{~m} \quad$ lehmiger Schluff, sandig, glimmerhaltig, hell gelblich braun $(2,5 \mathrm{Y}$ $6 / 4)$, grau marmoriert, mit nadelkopfgroßen Fe-/Mn-Konkretionen

$0,8 \mathrm{~m} \quad$ Fein- bis Mittelsand, leicht feinkiesig, gelbbraun (10 YR 5/7), mit zunehmendem Feinkies-Gehalt übergehend in

0,5 m Mittelkies (Quarzkiese), leicht grob- und feinkiesig, stark sandig, leicht lehmig, dunkel gelbbraun (10 YR 4/6)

\subsection{Profilbeschreibung}

Die 21 Horizonte können in vier fazielle Großeinheiten gegliedert werden (vgl. Abb. 2), eine untere Folge von Schluffen und fossilen Böden (Horizonte 11-20) über einem unteren sandigen Schotter (Horizont 21), darüber einen mächtigen Schwemmlehm mit zahlreichen Sand- und vereinzelten Kiesschnüren, der am Ostrand des Profils schon über ein Drittel der Gesamthöhe erreicht (Horizonte 9 und 10) und eine obere Folge von durch sechs Böden stark überprägten Schluffen, die, nur unmerklich nach NNE einfallend, über das gesamte Profil durchhält (Horizonte 1-8).

\subsubsection{Obere Folge}

Die jüngste fazielle Großeinheit ist eine vielgliedrige Schluff-Bodensequenz, die sich über der bedeutendsten Diskordanz des gesamten Profils entwickelte, dem sandig-kiesigen Umlagerungshorizont 9. Die Böden, Parabraunerden bzw. Pseudogley-Parabraunerden sind mehr oder weniger stark verkürzt und weisen außer dem rezenten Boden nur noch den Btbzw. den BtSd-Horizont auf. Die Basis dieser SchluffBodensequenz bildet ein Pedokomplex von drei denudativ verkürzten Pseudogley-Parabraunerden (2.-4. fBtSd), die durch scharfe Diskordanzen voneinander getrennt sind. Der Tonanreicherungshorizont des unteren dieser Böden (4. fBtSd) reicht bis in den liegenden Umlagerungshorizont 9 hinab. Lag die Mächtigkeit dieser Böden bei den Aufschlußverhältnissen während der Profilaufnahme (1984) bei $1,0-1,5 \mathrm{~m}$, ist infolge des fortgeschrittenen Abbaues der allerdings immer noch gekappte 4 . fBtSd nun in einer Mächtigkeit von rund $2 \mathrm{~m}$ aufgeschlossen, wobei er die liegenden Umlagerungshorizonte 9 und 10 , unter die Diskordanz hinabgreifend, bis in eine Tiefe von $1,5 \mathrm{~m}$ pedogen überprägt. Analog dürften die Mindestmächtigkeiten der beiden jüngeren Böden dieses Pedokomplexes ebenfalls rund $2 \mathrm{~m}$ betragen haben, ist doch auch der ebenfalls gekappte 1. $\mathrm{fBt}$ in einer Mächtigkeit von rund 1,9 m erhalten, während die rezente Parabraunerde mit ihrer Gesamtmächtigkeit von $0,7 \mathrm{~m}$, wovon nur $0,3-0,4 \mathrm{~m}$ auf den BtHorizont entfallen, auffallend geringmächtig ist. $\mathrm{Da}$ dies den Befunden in der näheren Umgebung entspricht, ist mit einer anthropogen bedingten flächenhaften Profilverkürzung zu rechnen, über die an anderer Stelle berichtet werden soll. Der maximale Tongehalt in den drei Bt-Horizonten des Pedokomplexes ist mit Werten zwischen 40 und $45 \%$ annähernd gleich hoch und entspricht damit dem des 1 . fBt und des Bt-Horizontes der rezenten Parabraunerde.

Über diesem Pedokomplex (2.-4. fBtSd) folgt ein schluffiger Lehm (Horizont 5), dessen obere zwei Drittel von einer Bodenbildung überprägt worden sind $(1 . \mathrm{fBt})$, die in einen hangenden $\mathrm{fBt}$ - und einen liegenden $\mathrm{fBv}$-Horizont differenziert werden kann. Der fBt-Horizont führt an seiner Basis vereinzelte Lößkindel.

Die größte Differenzierung weist das Profil über der jüngsten fossilen Parabraunerde $(1 \mathrm{fBt})$ auf. Diese wird von einem schluffigen Lehm (Horizont $4 \mathrm{a}$ ) überlagert, der zahlreiche Holzkohleflitter und -stückchen an seiner Basis führt, wobei dessen schwache Pseudovergleyung bis $0,2 \mathrm{~m}$ tief in den liegenden Bt-Horizont hinabgreift, der zudem in diesem Bereich eine von oben erfolgte Einschlämmung von Schluff auf den Gefügeflächen aufweist. Im schluffigen Lehm treten vereinzelte Frostspalten bis 0,6 m Länge auf, die bis in den liegenden Bt-Horizont hinabreichen. Der Lehm ist solifluidal verlagert, nachgezeichnet durch die fahnenartig in Gefällsrichtung ausgezogenen Frostspalten. Davon sind auch noch die oberen $25 \mathrm{~cm}$ des liegenden Bt-Horizontes betroffen. Über dem Lehm folgt ein liegender, nadelstichporiger Löß (Horizont $3 \mathrm{~b}$ ), der im Profil nur im Ostteil erhalten ist, in der weiteren Umgebung aber durchgehend verbreitet auftritt (vgl. Abb. 6). Vom bis $0,8 \mathrm{~m}$ mächtigen hangenden Löß (Horizont 2), der mit $24 \%$ den höchsten Kalkgehalt des Profils aufweist, wird dieser durch einen $0,5 \mathrm{~m}$ mächtigen Verbraunungshorizont getrennt, der Kalkkonkretionen führt. Im hangenden Löß ist ein $15-20 \mathrm{~cm}$ mächtiger, schwach verlehmter 


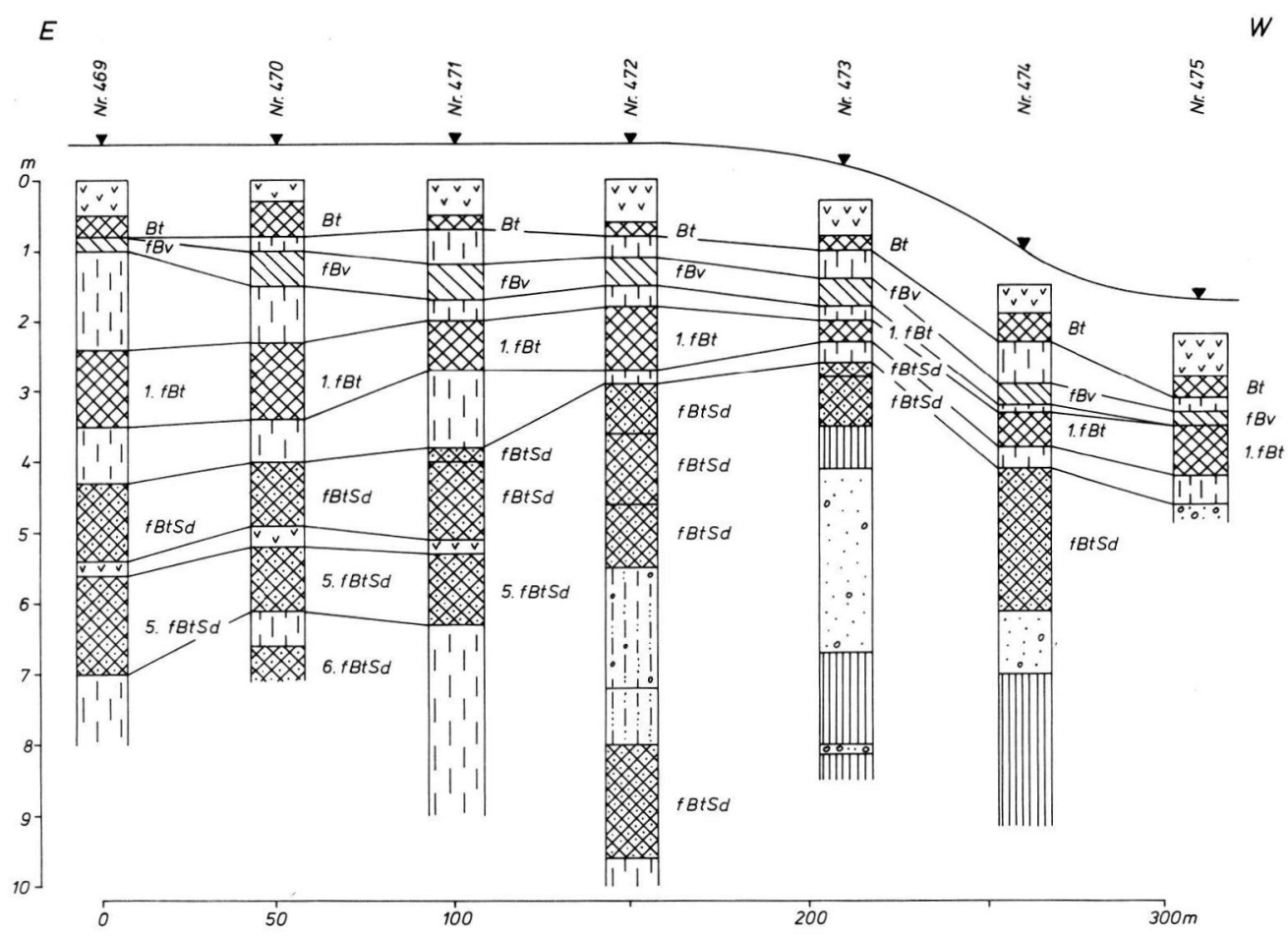

Abb. 6: Bohrprofiltreihe vom Westrand der Grube Hagelstadt nach W.

Das Profil Nr. 392 liegt ca. 20 m östlich der Profilreihe. Signaturlegende wie in Abb. 7.

graubrauner Horizont mit winzigen Rostflecken eingeschaltet, der zahlreiche Lößschnecken der Gattung Pupilla führt. Zudem konnten in diesem Horizont viele Knochenbruchstücke eines Großsäugers gefunden werden, die Herr Prof. Dr. GroIss (Institut für Paläontologie der Universität Erlangen) als Rhinocerus-Art bestimmte. Wegen des schlechten Erhaltungszustandes war jedoch keine nähere Bestimmung als Rhinocerus metatarsus oder Rhinocerus metacarpus möglich. Aufgrund der stratigraphischen Position der Funde in kaltzeitlichem, nur schwach verwitterten Löß kann aber wohl am ehesten an das Wollnashorn (Coelodonta antiquitatis) gedacht werden (HÜNERMANN 1987).

Das Profil wird von einer Parabraunerde (Horizont 1) abgeschlossen. Unter Fichtenhochwald ist sie in der gesamten Umgebung als podsolige Parabraunerde ausgebildet, wobei der Aeh-Horizont etwa $3 \mathrm{~cm}$ mächtig ist und Aeh- und Al-Horizont mit $0,4 \mathrm{~m}$ um $0,1 \mathrm{~m}$ mächtiger sind als der Bt-Horizont, der allerdings verbreitet bis $0,5 \mathrm{~m}$ taschen- und zapfenförmig in den liegenden Löß hinabgreift. Aufgrund der Tonarmut des Al-Horizontes und dessen Mächtigkeit von über 0,3 m könnte dieser auch als Ael-Horizont angesprochen werden und das Bodenprofil als Fahlerde (ARBEITSKREIS FÜR BODENSYSTEMATIK 1985: 39). Die
$\mathrm{pH}$-Werte dieser Parabraunerde liegen alle unter 4 , am niedrigsten im Aeh-Horizont mit 3,1 im Bt-Horizont bei 3,7 und kontrastieren damit stark zu den im gesamten Profil gemessenen Werten, die zwischen 6,3 und 7,5 liegen (vgl. Abb. 5).

\subsubsection{Umlagerungsfolge}

Die markanteste Diskordanz im Profil Hagelstadt markiert der Horizont 9, ein Umlagerungshorizont, der stratigraphisch tiefere Horizonte kappt und eine Dellenfüllung abschließt. Diese Delle wird von der fünften fossilen Parabraunerde (Horizont 12 und 13) nachgezeichnet (vgl. Abb. 2). Während Horizont 9 aus sand- und kiesstreifigem Schwemmlehm besteht, der durchgehend kleine Bruchstücke von Bodenaggregaten bis $2 \mathrm{~mm}$ Durchmesser führt und somit genetisch als Lehmbröckelsand (ROHDENBURG 1971: 310) angesprochen werden kann, weist der liegende Horizont 10 dieser Umlagerungsfolge zwar merklichen Sandgehalt, aber trotz erkennbarer Feinschichtung keine Sandstreifigkeit mehr auf. Genetisch bilden beide Horizonte eine Einheit, die aus Material aufgebaut wird, das am flachen Hang westlich der Delle und auf der angrenzenden Hochfläche erodiert wurde. Wird Horizont 10 aus umgelagertem Schluff 
aufgebaut, ist bei Horizont 9 auch umgelagertes Material der liegenden fossilen Böden (Horizonte 12 und tiefer) und Kies und Sand noch unbekannter Herkunft beteiligt. Während beide Horizonte zusammen mit der Gruben-Westwand nur wenige Dezimeter mächtig sind, nehmen sie zum Dellentiefsten hin, das bei der Profilaufnahme 1984 noch nicht aufgeschlossen war, auf über $8 \mathrm{~m}$ Gesamtmächtigkeit zu. Ein kräftiger Verbraunungshorizont (Horizont 11), der im Gesamtprofil (vgl. Abb. 2) an der Basis der Umlagerungsfolge ausgebildet ist und dort unmittelbar dem Al-Horizont der liegenden fünften fossilen Parabraunerde aufsitzt, war im 1987 kurzfristig aufgeschlossenen östlichen Grubenteil innerhalb des Schwemmlehmes (Horizont $10 \mathrm{~b}$ ) ausgebildet und wurde dort noch von über $1 \mathrm{~m}$ dieses Lehmes unterlagert.

\subsubsection{Liegendfolge}

Die liegende fazielle Großeinheit ist wie die obere Folge eine vielgliedrige Schluff-Bodensequenz mit drei fossilen Böden interglazialen Typs. Beim jüngsten dieser Böden (5. fBtSd), einer Pseudogley-Parabraunerde (Horizonte 12-13), die im Bereich des Profils sanft in eine Delle hinabzieht (vgl. Abb. 2), ist durchgehend noch der AlSw-Horizont (Horizont 12) mit einer mächtigen Konzentration von $\mathrm{Fe}$-/Mn-Konkretionen bis zu $2 \mathrm{~cm}$ Durchmesser an seiner Basis erhalten. Allerdings tritt er in Plateau- und Hanglagen nur erosiv verkürzt auf, während im Dellentiefsten seine Mächtigkeit durch die Zufuhr umgelagerten Materials auf fast 2,5 m ansteigt. Da die Konkretionen dieses Horizontes mit Abstand die größten im gesamten Profil sind, kann bei Bohrungen in der Umgebung aufgrund dieses Al-Horizontes und ihres Vorkommens darin auch bei unvollständigen Bodensequenzen der liegende $\mathrm{fBtSd}$-Horizont eindeutig als der 5. fBtSd identifiziert werden, wie dies in Abb. 6 dargestellt ist. Die Gesamtmächtigkeit des Bodens, der durch Verwitterung eines sandigen Lehmes (Horizont 14) entstanden ist, beträgt rund 3,5 $\mathrm{m}$ und liegt damit weit über den Mächtigkeiten vergleichbarer Böden der oberen Folge. Von diesen unterscheidet er sich auch deutlich durch seine auffallend gelblich-rote Farbe, während die Böden der oberen Folge und auch die im Liegenden braun bis kräftig braun sind. Von den beiden liegenden Böden ist der obere, ebenfalls eine Pseudogley-Parabraunerde (6. fBtSd, Horizont 15), die aus einem sandigen Lehm (Horizont 16) hervorging, durch Abtragung stark verkürzt, so daß vom BtSd-Horizont nur noch die basalen $0,6 \mathrm{~m}$ erhalten blieben, während die untere, siebte PseudogleyParabraunerde (Horizont 17), von der auch nur noch der BtSd-Horizont vorhanden ist, mit 3,4 m etwa der Gesamtmächtigkeit des nahezu vollständig erhaltenen fünften fBtSd entspricht. Aufgrund des hohen Anteils der Sandfraktion weist allerdings der BtSd-Horizont eine größere Vertikalerstreckung auf und kann wegen des Auftretens von vereinzelten Toninfiltrationsbändern als Übergangstyp zur Bänderparabraunerde angesprochen werden. Dieser mächtige Boden ging aus einer sandig-schluffigen Liegendfolge hervor (Horizonte 18-20), die stark hydromorph überprägt ist und aufgrund ihres auffallenden Gehaltes an hellen Glimmerflittern und der Sandstreifigkeit als Hochflutlehmsediment über dem liegenden sandigen Schotter anzusprechen ist.

\subsubsection{Unterer sandiger Schotter}

Dieser Schotter (Horizont 21) war nur durch eine Bohrung aufgeschlossen; er besteht aus Fein- und Mittelsand (Horizont 21a) mit einem zunehmenden Feinkiesgehalt und geht in liegenden Mittelkies (Horizont $21 \mathrm{~b}$ ) über. Neben Gangquarz kommen vereinzelte braune Hornsteine aus dem Weißen Jura, dunkelgraue und braune Lydite und quarzitische Sandsteine aus dem Saxothuringikum und rotbraune Radiolarite aus dem alpinen Einzugsgebiet der Donau vor. Verwitterungsanfälligere Gerölle aus Kalk, Granit und Gneis fehlen hingegen völlig.

\section{Zeitstellung und Diskussion}

Mit seinen acht Bt-Horizonten ist das Profil Hagelstadt zwar das zur Zeit vielgliedrigste Lößprofil Südbayerns, doch sind Profile mit der gleichen Anzahl oder sogar erheblich mehr Böden interglazialen Typs aus verschiedenen Regionen Mitteleuropas bekannt. So beschreiben SKOWRONEK \& WILLMANN (1984) die acht Bt-Horizonte des Profils Kirchheim in Unterfranken, SEMmel (1973) führt die neun Bt-Horizonte des Profils Bad Soden am Taunus und KUKLA (1975) die elf Bodenkomplexe des Lößprofils am Červený Kopec (Roter Berg) bei Brno in der Tschechoslowakei an. Das aus dreizehn interglazialen Bodenkomplexen bestehende Profil Dreihausen in Nordhessen (SABELBERG et al. 1976) und die siebzehn Böden interglazialen Typs im Profil Kremser Schießstätte im österreichischen Donauraum (FINK \& KUKLA 1977), an die sich vermutlich stratigraphisch die drei noch älteren fossilen Böden des Profils Stranzendorf (FINK et al. 1976: 102) anschließen lassen, die nach paläomagnetischen Untersuchungen von Koč (1978) über die Matuyama-Epoche bis in die Gauss-Epoche und damit noch weiter ins Pliozän hinabreichen als die des Profils Krems, belegen für das Quartär eine Warmzeit-Kaltzeit-Gliederung, die weit über die klassische alpine (PENCK \& BRÜCKNER 1909) und auch deren Erweite- 
rung um Donau-Günz (EBERL 1930) und BiberDonau (SCHAEFER 1965) hinausgeht. Aufgrund paläobotanischer Befunde postulieren auch FRENZEL (1973; 1987) und MENKE \& BEHRE (1973) mindestens elf Kaltzeit-Warmzeit-Zyklen während des Quartärs.

Wenn man davon ausgeht, daß in Mitteleuropa Böden mit Illuvialhorizonten als warmzeitliche, interglaziale Bodenbildungen anzusehen sind, was allerdings nicht unumstritten ist (SABELBERG et al. 1974), könnten die acht Parabraunerden bzw. PseudogleyParabraunerden des Profils Hagelstadt acht GlazialInterglazial-Zyklen des Quartärs repräsentieren. Allerdings sind alle diese Sediment-Boden-Zyklen unvollständig ausgebildet oder erhalten, weisen mehr oder minder große Schichtlücken auf oder sind polygenetische Bildungen, die von mehreren Warmzeiten überprägt wurden. Darauf weist ein Bohrprofil auf dem Plateau unmittelbar westlich Profil Nr. 392 hin (vgl. Abb. 6), wo in ausgedehnten Arealen zwischen dem ersten $\mathrm{fBt}$ und dem fünften $\mathrm{fBtSd}$ ein bis zwei Böden völlig fehlen. Aus den dargelegten Gründen muß bis zum Vorliegen weiterer Untersuchungsergebnisse auf eine Einordnung von sechs fossilen Böden interglazialen Typs des Profils Hagelstadt in die mitteleuropäische Quartärstratigraphie verzichtet werden.

\subsection{Unterer sandiger Schotter}

Ob der liegende Schotter als Typus des „Höhenhofer Schotters" (OsCHMANN 1958) angesprochen werden kann, was schon bei STRUNK (1989) diskutiert wurde, bleibt weiterhin offen. Dieser pleistozäne Schotter ist durch das Fehlen von instabilen Geröllen als Residualschotter mit sandig-lehmigem Zwischenmittel geröllpetrographisch kaum von den altpleistozänen Deckenschottern der Donau zu unterscheiden, die im Engtal zwischen Regensburg und Weltenburg in gleicher Höhenlage vorkommen (BAUBERGER, CRAMER \& TILLMANN 1969: 172; TillmanNs 1977: 107). Zudem taucht der Höhenhofer Schotter am Nordrand des Tertiärhügellandes zum Straubinger Becken hin unter die quartären Deckschichten ab, so daß er im Untersuchungsgebiet durchaus in gleichhoher oder sogar tieferer Position als die altpleistozänen Deckenschotter liegen kann.

Das Schottervorkommen in Hagelstadt liegt jedenfalls unter acht Böden interglazialen Typs, womit ihm ein Mindestalter von über $800 \mathrm{ka}$ zukäme. Die paläomagnetische Bearbeitung des mit Hagelstadt vergleichbaren Profils der Ziegeleigrube Strobel im benachbarten Regensburg durch BRUNNACKER et al. (1976) ergab für zwei Pseudogleye (Paläoböden 5 und 6) an der Basis des Profils über dem liegenden Schotter
(BRUNNACKER 1964) deren Zugehörigkeit zur Matuyama-Epoche. Im tiefsten Teil dieses Profils konnten BRUNNACKER et al. (1976) ein Alternieren von normaler und reverser Magnetisierung feststellen, die BRUNNACKER (1982) als Jaramillo-Event anspricht, wodurch die beiden Pseudogleye und der liegende Schotter des Profils ins Ältestpleistozän (0,9-1,2 Ma BP) hinabrückten.

\subsection{Jungpleistozän}

Im Vergleich zu den Profilen des Jungpleistozäns in Nordwestdeutschland (ROHDENBURG \& MEYER 1979; WALTHER \& BROSCHE 1982) oder Hessen (SEMMEL 1968) sind diejenigen des Regensburger Raumes ärmer an interstadialen Bodenbildungen. Klimatisch liegt dieses Profil noch im Trockengebiet der Regensburger Bucht mit Jahresmitteln des Niederschlags um $650 \mathrm{~mm}$ und damit nach BRUNNACKER (1957) im nördlichsten, trockensten Bezirk der würmzeitlichen Lößfazies Südbayerns. Verglichen mit der Lößfazies im benachbarten Österreich mit seinem bereits merklich kontinentaleren Klima ist der Regensburger Raum hingegen in die „Feuchte Lößlandschaft” (FINK 1979) einzuordnen, die sich von der dortigen „Trockenen Lößlandschaft" durch das Auftreten eines Naßbodens (Gleyfleckenzone) im Jungwürm-Löß über dem Verbraunungshorizont des Stillfried BInterstadials unterscheidet.

Der detaillierte pedologische Aufbau des Jungpleistozäns von Hagelstadt ist in Abb. 7 dargestellt. Das Liegende bildet der Bt-Horizont der Parabraunerde des Riß-/Würm-Interglazials. Zwei Thermolumineszenz-Datierungen im entkalkten Lößlehm an der Basis dieses Bodens ergaben inzwischen $138 \pm 14 \mathrm{ka}$ und $141 \pm 16 \mathrm{ka} \mathrm{BP}$ (BUCH \& ZÖLLER 1990). Darüber folgt ein vergleyter schluffiger Lehm, der an der Basis reichlich Holzkohleflitter führt. Diesen Lehm, BRUNNACKER (1957) spricht ihn als Basisfließerde des Würm-Frühglazials an, bezeichnet ROHDENBURG (1971: 297) als „Bilshäuser Bodenkomplex”, eine eigenständige Bodenbildung des Altwürm. Die stratigraphische Grenze zwischen Riß und Würm liegt deshalb nach ROHDENBURG \& MEYER (1979: 17) an der Oberkante des interglazialen Bt-Horizontes, dessen obere Dezimeter durch die altwürmzeitliche Bodenbildung noch sekundär pseudovergleyt sein können, wie dies auch im Profil Hagelstadt der Fall ist. Der Altwürm-Boden ist schwach solifluidal verlagert. Diese Basisfließerde wurde mit einem TL-Alter von $74 \pm 7,4$ ka BP datiert (BUCH \& ZÖLLER 1990). Die drei altwürmzeitlichen „Mosbacher Humuszonen" (SEMMEL 1968: 13), die in den pollenanalytisch nachgewiesenen Interstadialen Amersfoort, Brørup (ZAGWIJN 1961; DÜCKER 1967) und Odderade (DÜCKER 


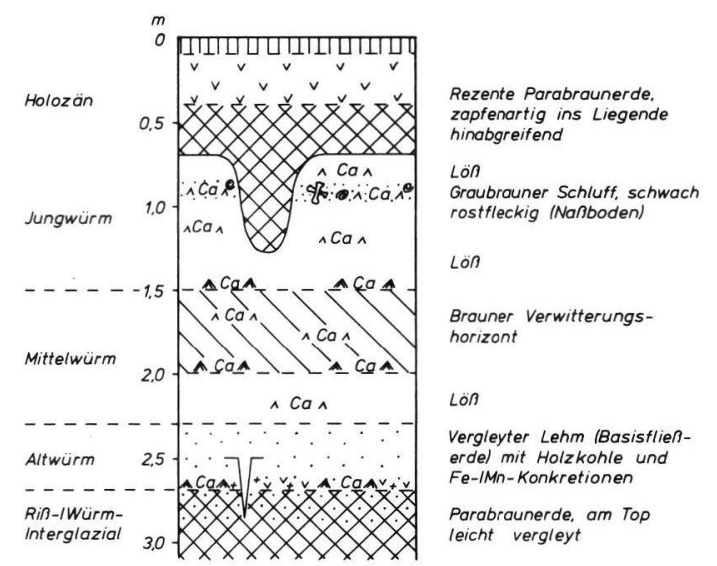

\section{GEOLOGIE \\ पI 1100
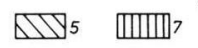 \\ PEDOLOGIE \\ सx.2 민 \\ $2 x_{6}$ \\ $v^{v} v^{v} 8$

Abb. 7: Pedologische Gliederung des Jungpleistozäns im Profil Hagelstadt. Signaturlegenden der geologischen und pedologischen Profile: 1 = schluffig äolisch;

2 = schluffig/schluffig-sandig (kolluvial);

3 = sandig/kiesig (kolluvial/fluvial); 4 = tonig;

5 = Bv-Horizont; 6 = Bt-Horizont; 7 = humos; 8 = Al-Horizont; 9 = vergleyt;

$10=$ Lößschnecken $/$ Knochen; $11=\mathrm{CaCO}_{3}$-Illuviation; 12 = Lößkindel; 13 = Frostspalte $/$ Holzkohle.

\& HUMMEL 1967) entstanden und von BRUNNACKER (1970) und SEMMEL \& STÄBLEIN (1971) aus dem ähnlich trockenen nordbayerischen Franken beschrieben wurden, fehlen hingegen in Hagelstadt wie im Regensburger Raum. Auch ein Verbraunungs- und Verlehmungshorizont, wie ihn ROHDENBURG \& MEYER (1979: 17) aus Südniedersachsen und Nordhessen als "Niedervellmarer Bodenkomplex" beschreiben, ist nicht vorhanden. Vielmehr wird die Basisfließerde des Altwürm von Löß überlagert, der von einer Verbraunung überprägt wird. Sie entspricht dem „Braunen Verwitterungshorizont", den BRUNNACKER (1957) ins Mittelwürm stellt. Zweifellos handelt es sich um eine Bodenbildung interstadialen Typs, die mit der des Stillfried B-Interstadials im benachbarten österreichischen Donauraum (FINK 1956) oder mit der entsprechenden Bodenbildung in pleistozänen Lössen der Tschechoslowakei (KLIMA et al. 1962) parallelisiert werden kann und somit stratigraphisch dem „Lohner Boden" Nordhessens (SCHÖNHALS, ROHDENBURG \& SEMMEL 1964; ROHDENBURG \& MEYER 1979) entspräche. Die weite Verbreitung dieses Bodens in Mitteleuropa stellte zuletzt RICKEN (1983: 129) zusammenfassend dar. Allerdings ist er im Regensburger Raum aus dem Hangendlöß sekundär aufgekalkt worden. ZAGWIJN \& PAEPE (1968) halten diesen Boden

für eine Bildung des Denekamp-Interstadials, das sie auf 29-35 ka BP und BURACZYNSKI \& BUTRYM (1987) auf $28-32 \mathrm{ka}$ BP datieren.

Die Jungwürm-Abfolge ist in Hagelstadt vergleichsweise wenig differenziert. Dort tritt ein typischer Löß mit einem schwach entwickelten Naßboden im oberen Drittel auf, den ZÖLLER mit der TL-Methode auf $25,1 \pm 2,3$ ka BP datierte (BUCH \& ZÖLLER 1990). Da der Regensburger Raum aber bereits außerhalb des Verbreitungsgebietes des Eltviller Tuffes liegt (MEIJS et al. 1983: Fig. 2), ist die stratigraphische Zuordnung des Naßbodens von Hagelstadt zu einem der fünf Jungwürm-Böden Hessens und des Rhein-Main-Gebietes (BIBUS \& SEMMEL 1977) vorerst nicht möglich.

\section{Dank}

Der Autor dankt Prof. Dr. Heine und Dr. BuCH, Regensburg, Prof. Dr. Petersen, Dr. Jerz und Dr. DREXIER, München und Dr. HAVLICEK, Prag, für kritische Diskussionen im Gelände, ebenso den Teilnehmern der Exkursionstagung 1988 des AK Paläoböden der Deutschen Bodenkundlichen Gesellschaft. Dr. ZÖLLER, Heidelberg, sei für die TL-Datierungen gedankt. Die Knochenfunde im Jungwürm-Löß verdanke ich meinem Sohn Clemens. Herr Prof. Dr. GROISS, Erlangen, war so freundlich, deren Bestimmung vorzunehmen. Nicht zuletzt sei Frau BERIE, Frl. SANCHEZ, Dr. BuCH und den Herren HilgarT und KUFELD für ihre Unterstützung bei den Profilaufnahmen und im Labor gedankt.

\section{Schriftenverzeichnis}

ARbeITSKReIS FÜr BODENSYSTEMATIK DER DeUTSCHEN BODENKUNDLICHEN GESELLSCHAFT (Hrsg.) (1985): Systematik der Böden der Bundesrepublik Deutschland. - Mitt. Dt. Bodenkundl. Ges., 44: 1-90; Hannover.

Bauberger, W., Cramer, P. \& Tillmann, H. (1969): Erläuterungen zur Geologischen Karte von Bayern 1: 25000 , Blatt Nr. 6938 Regensburg, 414 S.; München (Bayer. Geol. L.-Amt).

Bibus, E. \& Semmel, A. (1977): Stratigraphische Leithorizonte im Würmlöß des Mittelrheingebietes. - Geol. Jb. Hessen, 105: 141-147; Wiesbaden.

BrunNaCKer, K. (1957): Die Geschichte der Böden im jüngeren Pleistozän in Bayern. - Geologica Bavarica, 34: 1-95; München.

- (1964): Böden des älteren Pleistozäns bei Regensburg. - Geologica Bavarica, 53: 148-160; München.

- (1970): Zwei Lößprofile extremer Klimabereiche Bayerns. - Geologica Bavarica, 63: 195-206; München. 
BRUNNACKER, K. (1982): Äolische Deckschichten und deren fossile Böden im Periglazialbereich Bayerns. Geol. Jb., F 14: 15-25; Hannover.

—, Boenigk, W., KočI, A. \& Tillmanns, W. (1976): Die Matuyama/Brunhes-Grenze am Rhein und an der Donau. - N. Jb. Geol. u. Paläont., Abh., 151: 358-378; Stuttgart.

BUCH, M. W. \& ZÖLLER, L. (1990): Gliederung und Thermolumineszenz-Chronologie der Würmlösse im Raum Regensburg. - Eiszeitalter u. Gegenwart, 40: 63-84; Hannover.

BURACZYNSKI, J. \& BUTRYM, J. (1987): Thermoluminescence stratigraphy of the loess in the Southern Rhinegraben. - Catena Suppl., 9: 81-94; Braunschweig.

DUUCKER, A. (1967): Interstadiale Bodenbildungen als stratigraphische Zeitmarken im Ablauf der Weichselkaltzeit in Schleswig-Holstein. - Fundamenta, B 2: 30-73; Köln.

— \& Hummel, P. (1967): Die fossilen Böden von Odderade/Dithmarschen, ein Beitrag zur Stratigraphie der Weichselkaltzeit. - Fundamenta, B 2: 80-100; Köln.

EBERL, B. (1930): Die Eiszeitenfolge im nördlichen Alpenvorlande. Ihr Ablauf, ihre Chronologie auf Grund der Aufnahme im Bereich des Lech- und Illergletschers: 427 S.; Augsburg (Filser).

FINK, J. (1956): Zur Korrelation der Terrassen und Lösse in Österreich. - Eiszeitalter u. Gegenwart, 7: 49-77; Öhringen.

- (1979): Stand und Aufgaben der österreichischen Quartärforschung. - Innsbrucker Geogr. Studien, 5 (Leidlmair-Festschrift): 79-104; Innsbruck.

-, Fischer, H., Klaus, W., KoČI, A., KoHl, H., KuKLA, J., LOŽEK, V., PIFFL, L. \& RABEDER, G. (1976): Exkursion durch den österreichischen Teil des nördlichen Alpenvorlandes zwischen Krems und Wiener Pforte. Mitt. d. Kommission f. Quartärforsch. d. Österr. Akad. d. Wiss., 1: 1-113; Wien.

- \& KuKLA, G. J. (1977): Pleistocene climates in central Europe: At least 17 interglacials after the Olduvai Event. - Quaternary Research, 7: 363-371; New York.

Frenzel, B. (1973): 3. On the Pleistocene vegetation history. - In: SCHÖNHALS, E. \& HUCKRIEDE, R. (eds.): State of research on the Quaternary of the Federal Republic of Germany: C. Area between the Scandinavian and the Alpine glaciation. - Eiszeitalter $u$. Gegenwart, 23/24: 321-332; Öhringen.

- (1987): Grundprobleme der Vegetationsgeschichte Mitteleuropas während des Eiszeitalters. - Mitt. d. Naturforsch. Ges. Luzern, 29: 99-122; Luzern.

Homilus, J., Weinig, H., Brost, E. \& BADER, K. (1983): Geologische und geophysikalische Untersuchungen im Donauquartär zwischen Ulm und Passau. - Geol. Jb., E 25: 73 S.; Hannover.

HÜNERMANN, K. (1987): Faunenentwicklung im Quartär. - Mitt. d. Naturforsch. Ges. Luzern, 29: 151-171; Luzern.
Kuima, B., Kukla, J., LožeK, V. \& DE Vries, H. (1962): Stratigraphie des Pleistozäns und Alter des paläolithischen Rastplatzes in der Ziegelei von Dolní Věstonice (Unter-Wisternitz). - Anthropozoikum, 11: 93-145; Prag.

KočI, A. (1978): Paleomagnetic research of Quaternary sediments at Central European localities. - In: NAGL, H. (Hrsg.): Beiträge zur Quartär- und Landschaftsforschung (Fink-Festschrift), 261-267; Wien.

KUKLA, G. J. (1975): Loess stratigraphy of Central Europe. - In: Butzer, K. W. \& ISAAC, G. L. (eds.): After the Australopithecines, 99-188; The Hague, Paris (Mouton).

LEgER, M. (1965): Les terrasses du Danube de Regensburg à Pleinting. - Bull. Ass. française pour l'Etude du Quaternaire, 2: 153-164; Paris.

Meijs, E., MÜcher, H., Ouwekerk, G., Romein, A. \& STOLTENBERG, H. (1983): Evidence of presence of the Eltville tuff layer in Dutch and Belgian Limbourg and the consequences for the loess stratigraphy. - Eiszeitalter u. Gegenwart, 33: 59-78; Hannover.

MenKe, B. \& Behre, K. E. (1973): 2. History of vegetation and biostratigraphy. - In: SCHÖNHALS, E. \& HUCKRIEDE, R. (eds.): State of research of the Quaternary of the Federal Republic of Germany, A. Area of the Scandinavian Glaciation. - Eiszeitalter u. Gegenwart, 23/24: 251-267; Öhringen.

OschmanN, F. (1958): Erläuterungen zur Geologischen Karte von Bayern 1: 25000, Blatt Nr. 7038 Bad Abbach, 184 S.; München (Bayer. Geol. L.-Amt).

Penck, A. \& BrüCKner, E. (1909): Die Alpen im Eiszeitalter, 3 Bde., 1199 S., 350 Taf., 19 Ktn.; Leipzig (Tauchnitz).

RICKEN, W. (1983): Mittel- und jungpleistozäne Lößdecken im südwestlichen Harzvorland. - Catena, Suppl., 3: 95-138; Braunschweig.

ROHDENBURG, H. (1971): Einführung in die klimagenetische Geomorphologie, 2. Aufl.: 30 S.; Gießen (Lenz).

— \& MeYer, B. (1979): Zur Feinstratigraphie und Paläopedologie des Jungpleistozäns nach Untersuchungen an südniedersächsischen und nordhessischen Lößprofilen. - Landschaftsgenese und Landschaftsökologie, 3: 1-89; Braunschweig.

Sabelberg, U., Mavrocordat, G., Rohdenburg, H. \& SCHÖNHALS, E. (1976): Quartärgliederung und Aufbau von Warmzeit-Kaltzeit-Zyklen in Bereichen mit Dominanz periglazialer Hangsedimente, dargestellt am Quartärprofil Dreihausen/Hessen. - Eiszeitalter u. Gegenwart, 27: 93-120; Öhringen.

-, RoHDenburg, H. \& HAVelBerg, G. (1974): Bodenstratigraphische und geomorphologische Untersuchungen an den Lößprofilen Ostheim (Kreis Hanau) und Dreihausen (Kreis Marburg) und ihre Bedeutung für die Gliederung des Quartärs in Mitteleuropa. - RheinMainische Forschungen, 78: 101-120; Frankfurt. 
SCHAEFER, I. (1965): The succession of fluvioglacial deposits in the Northern Alpine Foreland. Proc. VII Congress INQUA, 14: 9-14; Boulder-Denver, Col.

Schönhals, E., Rohdenburg, H. \& Semmel, A. (1964): Ergebnisse neuerer Untersuchungen zur Würmlöß-Gliederung in Hessen. - Eiszeitalter u. Gegenwart, 15: 199-206; Öhringen.

SEMmel, A. (1968): Studien über den Verlauf jungpleistozäner Formung in Hessen. - Frankfurter Geogr. Hefte, 45: 133 S., Frankfurt.

- (1973): 1. Periglacial sediments and their stratigraphy. - In: SCHÖnhals, E. \& HuCKRIEDE, R. (eds.): State of research on the Quaternary of the Federal Republic of Germany: C. Area between the Scandinavian and the Alpine Glaciation. - Eiszeitalter u. Gegenwart, 23/24: 293-305; Öhringen.

— \& STÄBLEIN, G. (1971): Zur Entwicklung quartärer Hohlformen in Franken. - Eiszeitalter u. Gegenwart, 22: 23-34; Öhringen.

SkowroneK, A. \& WillmanN, N. (1984): Ein reich gegliedertes Quartärprofil nördlich Kirchheim in Unterfranken. - Naturhist. Ges. Nürnberg, Jahresmitt., 1984: 105-107; Nürnberg.

StRUNK, H. (1989): Aspects of the Quaternary in the Tertiary Hills of Bavaria. - Catena, Suppl. 15: 289-295; Cremlingen.
Tillmanns, W. (1977): Zur Geschichte von Urmain und Urdonau zwischen Bamberg, Neuburg/Donau und Regensburg. - Sonderveröff. Geol. Inst. Universität Köln, 30: 198 S.; Köln.

WALTHER, M. \& BRosche, K.-U. (1982): Zur Bedeutung der Lößstratigraphie für die Rekonstruktion des jungpleistozänen Klimas im nördlichen Mitteleuropa am Beispiel norddeutscher Lößprofile. - Ber. d. naturhist. Ges. Hannover, 125: 97-159; Hannover.

WeINIG, H. (1980): Hydrogeologie des Donautales. - In: BAYERISCHES GEOLOGISCHES LANDESAMT (Hrsg.): Wasserwirtschaftliche Rahmenuntersuchung Donau und Main, 9-26; München.

ZAGWIJN, W. H. (1961): Vegetation, climate and radio carbon datings in the Late Pleistocene of The Netherlands. Part I: Eemian and early Weichselian. - Medd. van de Geol. Stichting, N.S., 14: 15-45; s'Gravenhage.

- \& PAEPE, R. (1968): Die Stratigraphie der weichselzeitlichen Ablagerungen der Niederlande und Belgiens. Eiszeitalter u. Gegenwart, 19: 129-146; Öhringen.

Manuskript eingegangen am 16.1.1989, Nachträge September 1989. 